\title{
Cosmological dark fluid from five-dimensional vacuum
}

\author{
Luz M. Reyes * José Edgar Madriz Aguilar \\ Departamento de Física, DCI, Campus León, Universidad de Guanajuato, C.P. 37150, León, Guanajuato, México.
}

(Dated: June 27, 2018)

\begin{abstract}
In the framework of the induced matter theory of gravity, we derive a 5D solution of the field equations that can describe a 4D cosmological scenario where the dark fluid (dark matter plus dark energy) equation of state has a geometrical origin. There is not a natural separation of the dark sector into different components, and then the model may provide a geometrical explanation for the existence of a dark degeneracy in cosmological scenarios.
\end{abstract}

PACS numbers: 04.50.-h,95.35.+d,95.36.+x

\section{INTRODUCTION}

The idea of the existence of exotic components in the universe, namely dark matter and dark energy, comes basically from three observational evidences: supernovae of the type Ia, surveys of clusters of galaxies, and observations of anisotropies in the Cosmic Microwave Background Radiation (CMBR) [1]. Observations of high redshift of type Ia supernovae suggest that the expansion of the universe is accelerating [2 4]. Surveys of clusters of galaxies indicate that the energy density of matter is less than the critical density of the Universe [5], whereas observations of the temperature anisotropies of the Cosmic Microwave Background (CMB) show evidence that our Universe is spatially flat, and then the total energy density parameter is very close to the critical one [6].

In obtaining these observational constraints, it has been considered the model assumption that there are two separated dark components: dark matter, responsible for the formation of cosmological structure, and dark energy, responsible for the accelerated expansion of the Universe. From this arises the so-called standard cosmological model, also known as $\Lambda \mathrm{CDM}$, where dark matter is a (cold) pressureless fluid and the dark energy is described by a cosmological constant [1, 6 ].

However, the observations from which these two components have been detected are gravitational in nature, and these kind of measurements are unable to provide information about a unique decomposition of the dark sector into these components[7]. As it was clearly argued in [8], when we are in a state of total ignorance about the nature of a single one of the dark components, we can also not completely measure the others.

In this case, the separation into dark matter and dark energy can be seen as a convenient parametrization without experimental reality. In models where this split is assumed, it is necessary to impose some additional conditions upon the models in order to make the assumption

\footnotetext{
* luzreyes@fisica.ugto.mx

$\dagger$ jemadriz@fisica.ugto.mx

$\ddagger$ lurena@fisica.ugto.mx
}

well-defined. These conditions can be that the dark energy vanishes at high redshift, or that the dark energy constitutes the non-clustering part of the dark energymomentum tensor. The lack of guidance of gravity observations leads to the so-called dark degeneracy [8].

Theoretically, a great effort has been done to construct models to explain dark energy. In the literature, we can find proposals, among others, like quintessence models, interacting models of dark energy, k-essence models and proposals coming from modified theories of gravity [9], see also $10-12]$ and references there in. Other alternatives are models based in theories with extra dimensions, like the induced matter (IM) theory of gravity [13, 14] and Brane World (BW) scenarios [15].

In $\mathrm{BW}$ scenarios, the $4 \mathrm{D}$ universe is viewed as a hypersurface called the brane, which is embedded in a higher dimensional spacetime called the bulk. In this context, ordinary matter is confined to the brane by a variety of different mechanisms, while gravity can propagate freely through the bulk.

Parallel to BW scenarios we have the IM theory. This theory can be considered as an extension of $4 \mathrm{D}$ general relativity to 5D. In this approach, our Universe is described by a $4 \mathrm{D}$ hypersurface embedded in a $5 \mathrm{D}$ Ricciflat $\left({ }^{(5)} R_{a b}=0\right)$ spacetime. The extra dimension is considered as non-compact, and classical sources of matter in $4 \mathrm{D}$ are identified with the curvature of the $4 \mathrm{D}$ hypersurface. This curvature is a consequence of the embedding [14].

The IM theory is mathematically supported by the Campbell-Magaard theorem, that states that any analytical solution of the $n$-dimensional Einstein equations can be embedded in a $(n+1)$-dimensional Ricci-flat manifold [16 18]. Both the BW and the IM theory have different physical motivations, an equivalence to each other has been shown by Ponce de Leon in [19]. In spite of such an equivalence, the requirement in IM theory of starting from a 5D Ricci-flat spacetime, makes the task of finding solutions easier than in the BW theories.

The purpose of this paper is to derive a particular solution of the 5D field equations of the IM theory capable to describe not just the dark energy component of the universe, but the full dark sector (dark matter + dark energy) as a single dark fluid component. An exact solu- 
tion is found from the expected behavior of the dark fluid equation of the state in the standard $\Lambda$ CDM model. The solution also unifies previous works in which dark matter and dark energy were studied separately in IM theory.

The paper is organized as follows. In Sec. III, we obtain the geometrical equation of state for the induced matter, from a class of 5D solutions, and we establish the effective $4 \mathrm{D}$ field equations. In Sec. III, we obtain a particular solution that describes, in the sense of induced matter, the $4 \mathrm{D}$ cosmological dark fluid. Finally, in Sec. IV] we give some final comments.

\section{5D SOLUTIONS AND 4D INDUCED MATTER}

Let us start by considering a 5D Ricci-flat spacetime $\left(\mathcal{M}^{5}, g\right)$. In order to describe neutral matter in the local coordinates $\left\{\xi^{a}\right\}=\left\{x^{\alpha}, \psi\right\}$, we choose a coordinate gauge that allows us to write the $5 \mathrm{D}$ line element in the form

$$
d S_{5}^{2}=g_{\alpha \beta}(x, \psi) d x^{\alpha} d x^{\beta}+\epsilon g_{\psi \psi}(x, \psi) d \psi^{2},
$$

where $\psi$ is the space-like and non-compact extra coordinate, and parameter $\epsilon= \pm 1$ accounts for the signature of the extra coordinate. Our conventions are: Latin (Greek) indices take values $0,1,2,3,4(0,1,2,3)$, and the metric signature is $(+,-,-,-,-)$, and we use units in which $c=1$.

In IM theory the 4D field equations are in general constructed as follows [14. From the 5D field equations ${ }^{(5)} R_{a b}=0$, and by means of the Gauss-Codazzi-Ricci equations for the embedding, we write the conventional 4D Ricci tensor ${ }^{(4)} R_{\alpha \beta}$. With the help of ${ }^{(4)} R_{\alpha \beta}$ and the induced metric $h_{\alpha \beta}(x)=g_{\alpha \beta}\left(x, \psi_{0}\right)$ on a generic hypersurface $\Sigma: \psi=\psi_{0}=$ constant, we also write the $4 \mathrm{D}$ scalar of curvature, ${ }^{(4)} R$.

We can form the $4 \mathrm{D}$ Einstein tensor ${ }^{(4)} G_{\alpha \beta}={ }^{(4)} R_{\alpha \beta}-$ $(1 / 2)^{(4)} R h_{\alpha \beta}$, leaving all remaining terms grouped together to form an effective (induced) energy-momentum tensor $T_{\alpha \beta}^{(I M)}$. The resulting field equations are then of the form ${ }^{(4)} G_{\alpha \beta}=8 \pi G T_{\alpha \beta}^{(I M)}$, where the energymomentum tensor of induced matter is purely geometrical in nature, and has the explicit form [13, 14]

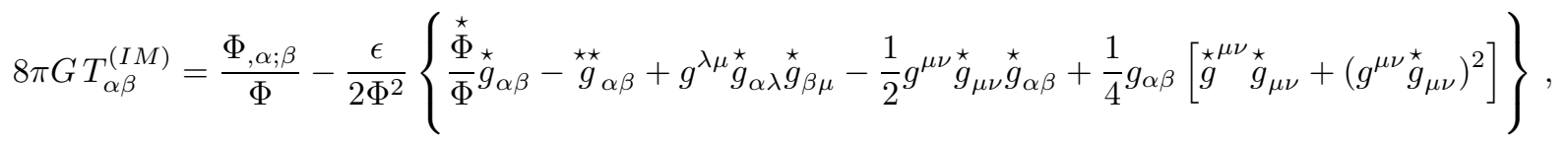

where $\Phi^{2}=g_{\psi \psi}$, the coma denotes partial derivative, the semicolon denotes 4D covariant derivative, and the star

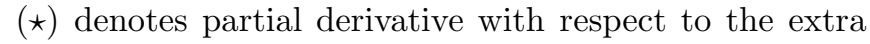
coordinate $\psi$.

In order to derive a cosmological scenario in which a combined dark fluid may be described, let us use the class of $5 \mathrm{D}$ solutions 20 22]

$d S_{5}^{2}=\left[\dot{A}^{2}(t, \psi) / \mu^{2}(t)\right] d t^{2}-A^{2}(t, \psi)\left(d r^{2}+r^{2} d \Omega^{2}\right)-d \psi^{2}$,

with

$$
A^{2}(t, \psi)=\left[\mu(t)^{2}+k\right] \psi^{2}+2 \nu(t) \psi+\frac{\nu(t)^{2}+K}{\mu(t)^{2}+k}
$$

where the dot denotes derivative with respect to the timelike coordinate $t, \mu(t)$ and $\nu(t)$ are arbitrary metric functions, and $k$ is the $3 \mathrm{D}$ curvature constant. Constant $K$ is related to the Kretschmann scalar, namely,

$$
I=R_{a b c d} R^{a b c d}=\frac{72 K^{2}}{A(t, \psi)^{8}}
$$

which shows that a non-null $K$ determines the Riemannian curvature of the 5D manifold [23].

Assuming that the 5D spacetime can be foliated by a family of hypersurfaces $\Sigma$, defined by the equation $\psi=$ constant, the geometry of each hypersurface, say at $\psi=$ $\psi_{0}$, will be then determined by the induced metric

$$
d S_{\Sigma}^{2}=\left[\dot{A}^{2}\left(t, \psi_{0}\right) / \mu^{2}(t)\right] d t^{2}-A^{2}\left(t, \psi_{0}\right)\left(d r^{2}+r^{2} d \Omega^{2}\right) .
$$

The Friedmann-Robertson-Walker (FRW) metric for a 4D flat universe, as supported by the observational evidence [6],

$$
d S_{\mid F R W}^{2}=d t^{2}-a^{2}(t)\left(d r^{2}+r^{2} d \Omega^{2}\right),
$$

where $a(t)$ is the scale factor of the Universe, can be recovered from the induced metric (6) by demanding the continuity of the metric across $\Sigma$. A comparison of Eqs. (6), and (7), indicate that the metric functions can be written as

$$
\begin{aligned}
& \mu(t)=a H, \\
& \nu(t)=-(a H)^{2} \psi_{0} \pm \sqrt{-K+a^{4} H^{2}},
\end{aligned}
$$

where $H=\dot{a} / a$ is the cosmological Hubble parameter, and we have set $k=0$. The double sign in Eq. (8b) appears because the Eqs. (4) and (8a) leave to a quadratic equation for the metric function $\nu$ when the continuity of the 5D metric is imposed. Physically, both solutions for $\nu$ induce the same energy density and pressure in $4 \mathrm{D}$ (see Eqs. (9)), leaving to the same 4D effective dynamics, and there is no dynamical reason to choose any sign. 
As it is usually done in the IM theory, after the identification of the induced matter on $\Sigma$ with a perfect fluid, we obtain, with the help of Eqs. (2), (3), and (8), that the total effective energy density and isotropic pressure, measured by $4 \mathrm{D}$ comoving observers, are given by [20]

$$
\begin{aligned}
& 8 \pi G \rho_{\text {eff }}={ }^{(4)} G_{0}^{0}=\frac{3 \mu^{2}}{a^{2}}, \\
& 8 \pi G p_{\text {eff }}=-{ }^{(4)} G_{1}^{1}=-\frac{2 \mu \dot{\mu}}{a \dot{a}}-\frac{\mu^{2}}{a^{2}},
\end{aligned}
$$

with ${ }^{(4)} G_{1}^{1}={ }^{(4)} G_{2}^{2}={ }^{(4)} G_{3}^{3}$. By means of Eqs. (8), and (9), we can define the effective equation of state

$$
w_{e f f} \equiv \frac{p_{e f f}}{\rho_{e f f}}=-\frac{1}{3}\left(1+2 \frac{\dot{\mu}}{\mu H}\right)=-\frac{1}{3}\left(1+2 \frac{\ddot{a} a}{\dot{a}^{2}}\right),
$$

which will give us information about the $4 \mathrm{D}$ physical sources of matter that are induced geometrically on the hypersurface modeling our $4 \mathrm{D}$ universe $\Sigma$. The last equality in Eq. (10) can be recognized as the typical one between the effective equation of state and the scale factor in a flat FRW Universe.

\section{INDUCING THE DARK FLUID}

In the $\Lambda \mathrm{CDM}$ model, the evolution of the total dark sector equation of state is given explicitly as

$$
w_{\text {dark }}(a) \equiv-\frac{\rho_{\Lambda}}{\rho_{\mathrm{CDM}}+\rho_{\Lambda}}=-\frac{a^{3}}{a^{3}+\alpha^{3}},
$$

where $\alpha=\sqrt[3]{\Omega_{\Lambda, 0} / \Omega_{\mathrm{CDM}, 0}}$ is the ratio of the present values of the dark energy and dark matter density parameters. In deriving Eq. (11), we have considered the convention for the normalization of the present value of the scale factor, $a_{0}=1$, that we will use hereafter.

In order to give a geometrical interpretation in the context of IM theory, of the dark fluid described by Eq. (11), we implement its formal identification with the geometrical equation of state, see Eq. (10), so that

$$
\frac{1}{3}\left(1+2 \frac{\ddot{a} a}{\dot{a}^{2}}\right)=\frac{a^{3}}{a^{3}+\alpha^{3}} .
$$

A full integration gives the known functional form of the scale factor $a(t)$ in a $\Lambda$ CDM model,

$$
a(t)=\alpha \sinh ^{2 / 3}\left[\frac{3}{2} \frac{H_{0} t}{\sqrt{1+\alpha^{3}}}\right] .
$$

This time, however, the matter contents only accounts for cold dark matter and the cosmological constant. The substitution of the result (13) into Eqs. (8), allows us to find the functional form of the metric functions,

$$
\begin{aligned}
\mu(t)= & \frac{\alpha^{5 / 2} H_{0}}{\sqrt{1+\alpha^{3}}}\left(\frac{\cosh \gamma(t)}{\sinh ^{1 / 3} \gamma(t)}\right), \\
\nu(t)= & -\frac{\alpha^{5} H_{0}^{2}}{1+\alpha^{3}}\left(\frac{\operatorname{coth}^{2} \gamma(t)}{\sinh ^{1 / 3} \gamma(t)}\right) \psi_{0} \\
& \pm \sqrt{\left.-K+\left(\frac{\alpha^{7 / 3} H_{0}^{2}}{1+\alpha^{3}}\right) \sinh ^{2 / 3} \gamma(t) \cosh ^{2} \gamma((t)) 4 \mathrm{~b}\right)}
\end{aligned}
$$

where we have used the auxiliary function $\gamma(t)=$ $(3 / 2)\left[H_{0} t / \sqrt{1+\alpha^{3}}\right]$.

Our results enclose some limiting cases already discussed in the literature. On one hand, when $H_{0} t \ll 1$, we find from Eq. (14a) that

$$
a(t) \propto t^{2 / 3} \Rightarrow \mu(t) \simeq \alpha\left(\frac{H_{0}}{\sqrt{1+\alpha^{3}}}\right)^{2 / 3}\left(\frac{3 t}{2}\right)^{-1 / 3}
$$

which corresponds to the cold 3D flat model studied within the IM framework in Ref. [20]. It can be seen that the effective equation of state, see Eq. (10), corresponds to dust, $p_{\text {eff }}=0$. Furthermore, we would like to point out that our solution gives a full integration of the general case discussed in 20] for a Universe containing dust and a cosmological constant.

On the other hand, when $H_{0} t \gg 1$, Eq. (14a) yields

$a(t) \approx \alpha \exp \left(\frac{H_{0} t}{\sqrt{1+\alpha^{3}}}\right) \Rightarrow \mu(t) \simeq \alpha H_{0} \exp \left(\frac{H_{0} t}{\sqrt{1+\alpha^{3}}}\right)$.

This limiting solution corresponds to a 3D-flat universe dominated by a cosmological constant, which has been extensively used in Refs. [22, 24] to derive inflationary scenarios from a non-compact Kaluza-Klein theory of gravity.

\section{FINAL COMMENTS}

We have studied, within the context of an IM theory of gravity, a cosmological scenario where the dark sector (dark matter plus dark energy) can be geometrically induced upon our $4 \mathrm{D}$ universe from a $5 \mathrm{D}$ Ricci-flat spacetime, as one single dark fluid.

We were able to find a complete solution of the $5 \mathrm{D}$ metric functions that covers both the matter and cosmological constant dominated epochs in the evolution of the Universe. The result contains, as limiting cases, solutions that have been derived and used by other authors also in the context of IM theory. The central point of the geometrical description relies on the formal identification of the geometrical equation of state with the physical one, whose functional form is given by the dynamics of the standard $\Lambda$ CDM model. It should be stressed out that only the dark matter and the dark energy components were considered. Other matter components, as photons, 
baryons, and neutrinos, are not part of the geometrical induced matter. However, these components can be included, if we extend our model to the context of hybrid models, in which the ideas of the IM theory and some ideas of brane cosmology can be combined. The idea of hybrid models has been introduced by J. Ponce de Leon in [19]. In these models the idea is to imposed the physics on the brane to restrict the freedom characteristic of many solutions in the IM theory. Hence, components like baryonic matter, neutrinos and photons could be introduced in the geometrical setting we are working on, in a similar way that it is done in brane cosmology, but this is matter of future investigation.

It can be seen from our results that the phenomenology associated to a single dark fluid, for which there is no natural separation between its components, can be given a geometrical interpretation from the point of view of a non-compact Kaluza-Klein theory of gravity. This fact may help to explain the existence of a dark degeneracy which is supported by (gravitational) observations.

\section{ACKNOWLEDGMENTS}

LMR and JEMA acknowledge support from CONACyT México. JEMA thanks the kind hospitality of the Departamento de Física of the Universidad de Guanajuato for a postdoctoral stay during which this work was initiated. This work was partially supported by PROMEP, DAIP, and by CONACyT México under grants 56946, and I0101/131/07 C-234/07 of the Instituto Avanzado de Cosmologia (IAC) collaboration.
[1] K. A. Olive, (2010), arXiv:1005.3955 [hep-ph],

[2] S. Perlmutter et al. (Supernova Cosmology Project), Astrophys.J. 517, 565 (1999), the Supernova Cosmology Project, arXiv:astro-ph/9812133 [astro-ph], Nature 391, 51 (1998), arXiv:astro-ph/9712212 [astro-ph] A. G. Riess et al. (Supernova Search Team), Astron.J. 116, 1009 (1998) arXiv:astro-ph/9805201 [astro-ph]; B. J. Barris, J. L. Tonry, S. Blondin, P. Challis, R. Chornock, et al., Astrophys.J. 602, 571 (2004), arXiv:astro-ph/0310843 [astro-ph].

[3] M. Sullivan, Lect.Notes Phys. 800, 59 (2010)

[4] B. Dilday et al. (SDSS Collaboration), $\quad$ Astrophys.J. 715, 1021 (2010), arXiv:1003.1521 [astro-ph.CO]; A. G. Riess, AIP Conf.Proc. 1166, 73 (2009).

[5] A. C. Pope et al. (The SDSS Collaboration), Astrophys.J. 607, 655 (2004), arXiv:astro-ph/0401249 [astro-ph].

[6] D. Larson, J. Dunkley, G. Hinshaw, E. Komatsu, M. Nolta, et al., (2010), arXiv:1001.4635 [astro-ph.CO], A. J. Benson, (2010), arXiv:1006.5394 [astro-ph.CO]

[7] M. Kunz, A. R. Liddle, D. Parkinson, and C. Gao, Phys.Rev. D80, 083533 (2009), arXiv:0908.3197 [astro-ph.CO]; W. Hu and D. J. Eisenstein, Phys.Rev. D59, 083509 (1999), arXiv:astro-ph/9809368 [astro-ph]; I. Wasserman, $\quad$ Phys.Rev. D66, 123511 (2002) arXiv:astro-ph/0203137 [astro-ph]; C. Rubano and P. Scudellaro, Gen.Rel.Grav. 34, 307 (2002), arXiv:astro-ph/0103335 [astro-ph]; A. R. Liddle and L. A. Urena-Lopez, Phys.Rev.Lett. 97, 161301 (2006), arXiv:astro-ph/0605205 [astro-ph].

[8] M. Kunz, $\quad$ Phys.Rev. D80, 123001 (2009) arXiv:astro-ph/0702615 [astro-ph].

[9] G. Caldera-Cabral, R. Maartens, and L. Urena-Lopez, Phys.Rev. D79, 063518 (2009), arXiv:0812.1827 [gr-qc] C. Armendariz-Picon, V. F. Mukhanov, and P. J. Steinhardt, Phys.Rev. D63, 103510 (2001) arXiv:astro-ph/0006373 [astro-ph]; S. Capozziello, S. Nojiri, and S. Odintsov, Phys.Lett. B634, 93 (2006) arXiv:hep-th/0512118 [hep-th]; R. Durrer and
R. Maartens, Gen.Rel.Grav. 40, 301 (2008) arXiv:0711.0077 [astro-ph]

[10] E. J. Copeland, M. Sami, and S. Tsujikawa, Int.J.Mod.Phys. D15, 1753 (2006), arXiv:hep-th/0603057 [hep-th],

[11] S. Tsujikawa, (2010), published in 'Dark Matter and Dark Energy: A Challenge for Modern Cosmology' Series: Astrophysics and Space Science Library, Vol. 370, ISBN: 978-90-481-8684-6, 2011, arXiv:1004.1493 [astro-ph.CO].

[12] D. Sapone, Int.J.Mod.Phys. A25, 5253 (2010) arXiv:1006.5694 [astro-ph.CO]

[13] J. Overduin and P. Wesson, Phys.Rept. 283, 303 (1997) arXiv:gr-qc/9805018 [gr-qc]

[14] P. S. Wesson, (1999), singapore, Singapore: World Scientific, 209 p; (2006), hackensack, USA: World Scientific, $222 \mathrm{p}$.

[15] I. Antoniadis, N. Arkani-Hamed, S. Dimopoulos, and G. Dvali, Phys.Lett. B436, 257 (1998) arXiv:hep-ph/9804398 [hep-ph]; N. ArkaniHamed, S. Dimopoulos, and G. Dvali, $\quad$ Phys.Lett. B429, 263 (1998) arXiv:hep-ph/9803315 [hep-ph]; L. Randall and R. Sundrum, Phys.Rev.Lett. 83, 3370 (1999), arXiv:hep-ph/9905221 [hep-ph]

Phys.Rev.Lett. 83, 4690 (1999)

arXiv:hep-th/9906064 [hep-th]; R. Maartens and K. Koyama, Living Rev. Rel. 13, 5 (2010), arXiv:1004.3962 [hep-th] A. Yazdani, K. Atazadeh, and S. Jalalzadeh, Int.J.Theor.Phys. 50, 888 (2011) P. Brax and C. van de Bruck, Class. Quant. Grav. 20, R201 (2003), arXiv:hep-th/0303095

[16] L. Magaard, (1963), phD dissertation; J. E. Campbell, (1926), book edited by Claredon Press.

[17] J. E. Lidsey, C. Romero, R. K. Tavakol, and S. Rippl, Class.Quant.Grav. 14, 865 (1997) arXiv:gr-qc/9907040 [gr-qc]

[18] C. Romero, R. K. Tavakol, and R. Zalaletdinov, Gen.Rel.Grav. 28, 365 (1996),

[19] J. Ponce De Leon, Mod.Phys.Lett. A16, 2291 (2001) arXiv:gr-qc/0111011 [gr-qc]

[20] H. Liu and P. S. Wesson, Astrophys.J. 562, 1 (2001) 
arXiv:gr-qc/0107093 [gr-qc]

[21] H.-Y. Liu and B. Mashhoon, Annalen Phys. 4, 565 (1995).

[22] J. Ponce De Leon, Gen.Rel.Grav. 20, 539 (1988)

[23] C.-W. Zhang, H.-Y. Liu, L.-X. Xu, and P. Wesson, Mod.Phys.Lett. A21, 571 (2006)
arXiv:astro-ph/0602414 [astro-ph]

[24] J. E. Madriz Aguilar and M. Bellini, $\quad$ Phys.Lett. B619, 208 (2005) arXiv:gr-qc/0503045 [gr-qc] J. E. Madriz Aguilar, Phys.Lett. B645, 6 (2007), arXiv:gr-qc/0611041 [gr-qc] 